\title{
An Extrinsic Fiber Fabry-Perot Interferometer for Dynamic Displacement Measurement
}

\author{
S. PULLTEAP ${ }^{1}$ and H. C. SEAT ${ }^{2}$ \\ ${ }^{1}$ Department of Mechanical Engineering, Faculty of Engineering and Industrial Technology, Silpakorn University \\ (Sanam Chandra Palace Campus), Nakhon Pathom, 73000, Thailand \\ ${ }^{2}$ Optoelectronics for Embedded Systems Group, INPT-ENSEEIHT, Rue Charles Camichel, BP $7122-31071$ Toulouse \\ Cedex7, France
}

${ }^{*}$ Corresponding author: S. PULLTEAP_ E-mail: saroj@su.ac.th

\begin{abstract}
A versatile fiber interferometer was proposed for high precision measurement. The sensor exploited a double-cavity within the unique sensing arm of an extrinsic-type fiber Fabry-Perot interferometer to produce the quadrature phase-shifted interference fringes. Interference signal processing was carried out using a modified zero-crossing (fringe) counting technique to demodulate two sets of fringes. The fiber interferometer has been successfully employed for dynamic displacement measurement under different displacement profiles over a range of $0.7 \mu \mathrm{m}$ to $140 \mu \mathrm{m}$. A dedicated computer incorporating the demodulation algorithm was next used to interpret these detected data as well as plot the displacement information with a resolution of $\lambda / 64$. A commercial displacement sensor was employed for comparison purposes with the experimental data obtained from the fiber interferometer as well as to gauge its performance, resulting in the maximum error of $2.8 \%$ over the entire displacement range studied.
\end{abstract}

Keywords: Fabry-Perot interferometer, vibration analysis, fringe counting technique, displacement profile

Citation: S. PULLTEAP and H. C. SEAT, “An Extrinsic Fiber Fabry-Perot Interferometer for Dynamic Displacement Measurement," Photonic Sensors, 2015, 5(1): 50-59.

\section{Introduction}

Fiber optic interferometers (FOIs), nowadays, are highly attractive instruments for high precision measurement especially for dynamic displacement measurement, mechanical vibration, and various other applications. It causes to several advantages such as the low weight and small size considerations, low power consumption, ruggedness, immunity to electromagnetic interference, and in particular, their extreme sensitivity and accuracy while being relatively unaffected by the target surface under test [1-3].
Basically, the FOIs can be classified into four main types: Michelson, Mach-Zehnder, Sagnac and Fabry-Perot interferometers, respectively. In the first to third interferometers, the light beam from a laser source is divided into two beams to generate reference and sensing signals with different optical paths. The combination of these two signals then results in an output interference signal as a consequence of the modulation of two cosinusoidal terms between the reference signal, due to reflection from an isolated fiber arm, and the sensing signal, which is obtained from back-reflection of a moving target. These devices have been frequently used for

Received: 2 July 2014 / Revised version: 20 November 2014

(C) The Author(s) 2014. This article is published with open access at Springerlink.com DOI: $10.1007 / \mathrm{s} 13320-014-0209-9$

Article type: Regular 
measuring strain, temperature, vibration, and acoustic analysis etc. [4].

The fiber-based Fabry-Perot interferometer (FFPI), on the other hand, is a latter type of the fiber sensor. Interference is generated from both the sensing and reference signals which propagate within a single fiber arm. Hence, no additional reference arm is required, leading to a reduction in optical components and hence cost savings when compared to either the Michelson or Mach-Zehnder interferometer. Moreover, the FFPI also exhibits superior performance in terms of the frequency bandwidth, sensitivity as well as precision [5-7], and has been frequently employed in velocity, strain, acoustics, temperature, micro-assembly process and vibration measurement [8-10].

In terms of displacement measurement investigation, several developed applications have been reported. For examples, X. Zhou et al. proposed a fiber-based Fabry-Perot interferometric sensor for high precision displacement measurement [11]. Fourier transformation combined with a signal processing method has been used for obtaining the sub-nanometer of the resolution over the entire measurement range. The developed sensor can thus be measured in the dynamic displacement range of $0 \mathrm{~mm}-3 \mathrm{~mm}$ with the resolution of $0.084 \mathrm{~nm}$. Moreover, a fiber optic sensor for two-dimension linear displacement measurement has, consequently, been described by A. Khiat et al. [12]. The displacement ranges of $8.67 \mathrm{~mm}$ and $13.03 \mathrm{~mm}$ have been investigated. The experimental results showed that the sensor can thus be exploited with the resolutions of $27.4 \mathrm{~nm}$ and also $38.7 \mathrm{~nm}$ over the entire displacement ranges.

As in all interferometers, the drawback of the characteristic cosinusoidal output signal can often lead to directional ambiguities in fringe motion when measurands such as the compressive/tensile stresses and strains, and vibration displace in counter directions [13]. To solve this problem, B. B. Chen et al. [14] proposed an introduction to a phase lead and lag in the interference signals (resulting in sine and cosine fringes with a quadrature phase-shift) generated by an electronic circuit to serve as the reference and sensing signals. From the phase relationship, when the sensing signal leads the reference signal, this corresponds to the target moving away from the sensing arm. If the sensing signal then lags the reference signal, this implies the target movement towards the sensor. However, the use of an electronic oscillator circuit can lead to a phase drift problem between the original interference signal and the synthetically generated phase-shifted component. To compensate for this effect, T. K. Gangopadhyay [15] introduced an interesting technique by modulating the drive current to the laser diode to obtain the phase lead and lag conditions in the interference signals in which the sensing signal was generated from the drive current variation to the laser source while the reference signal was obtained under no current modulation.

In recent work, an extrinsic fiber-based FabryPerot interferometric sensor has been developed for small displacement measurement without directional ambiguity [5]. In this work, we, however, exploited the fiber interferometer with a quadrature phase condition generated optically subject to three different excitation regimes (sinusoidal, triangular, and square waves) for investigating the dynamic displacement analysis. These waveforms were induced by a classical function generator at an excitation frequency of about $125 \mathrm{~Hz}$ but varied the amplitude for driving a mechanical vibrator. To obtain the displacement information, a demodulation program written in MATLAB was developed for plotting the displacement curve with a resolution of $\lambda / 64$ based on the modified zero-crossing fringe counting [16].

\section{Operating principles of fiber sensor}

\subsection{Dual-cavity generation fiber interferometer}

In general, the extrinsic fiber-based Fabry-Perot 
interferometer exploited here (EFFPI) uses the principle of a dual-cavity formed by the cleaved end of the fiber and the surface of the sensing element. With a reflection coefficient of the complete EFFPI measured at about $59 \%$, an estimated finesse of 9.5 can be obtained. The inherent configuration of the sensor may thus be approximated as a two-beam interferometer [17]. The operating principles of the fiber interferometer can therefore be described as follows: lightwave from a laser diode source is injected into the sensing arm via a $1 \times 2$ fiber coupler. Approximately $4 \%$ of the beam is reflected back from the fiber end as the reference beam $\left(I_{r}\right)$, while the rest is, consequently, transmitted to the target. The back-reflected beam from the target is re-injected into the fiber along the optical path as the sensing beam $\left(I_{s}\right)$. These two reflected beams thus interfere in the fiber from which a cosinusoidal signal is then generated at a photodetector with a phase-shift due to their optical path difference. This phenomenon is known as "interference" $(I)$ with a modulated intensity term given by

$$
I=I_{r}+I_{s}+2 \sqrt{I_{r} I_{s}} \cos \left(\theta_{r}-\theta_{s}\right)
$$

where $\left(\theta_{r}-\theta_{s}\right)$ is the phase difference between the reference and sensing beams, respectively, and is dependent on the variation of the cavity length so that, for normal incidence, any changes in the cavity length will lead to an optical path length change with a corresponding phase shift given by

$$
\Delta \theta=\theta_{r}-\theta_{s}=\frac{2 \pi n}{\lambda} 2 \Delta d
$$

with $n$ the refractive index of the sensing cavity $(\sim 1$ for air), $\lambda$ the injected wavelength, and $2 \Delta d$ the change in the cavity length for a Fabry-Perot interferometer (FPI).

For typical FPI signals, their cosinusoidal nature does not allow directional information on the target to be obtained directly unless additional processing schemes are employed. The following describes how the orthogonality of the fundamental mode was exploited in this proposed work to overcome directional ambiguity.

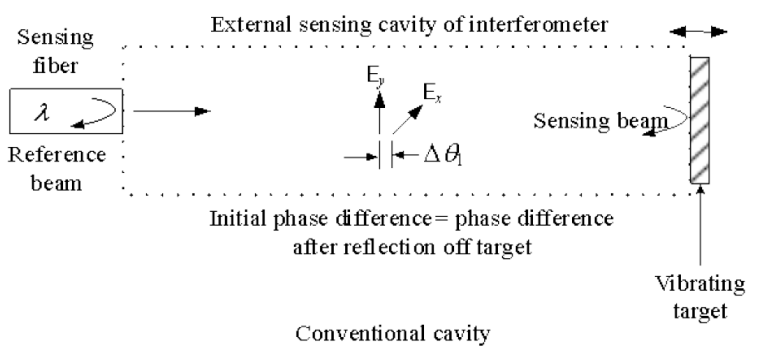

(a)

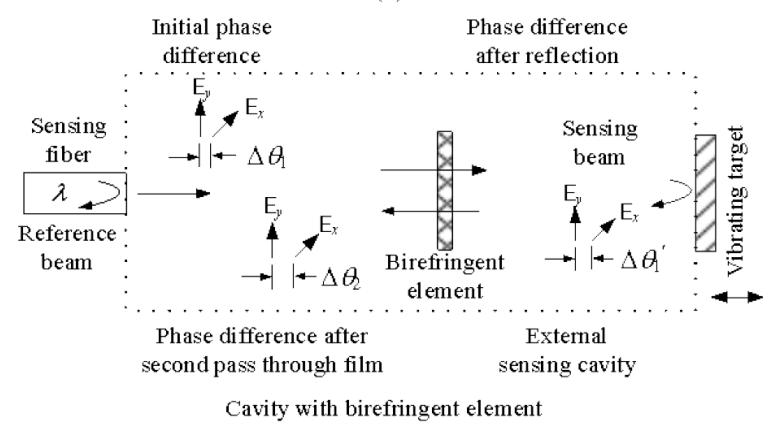

(b)

Fig. 1 Characteristics of the signal in the fiber interferometer: (a) "classical" Fabry-Perot external sensing cavity without the optical birefringent element in the optical path (phase retardation before and after reflection from the target remains unchanged and (b) introduction to the optical birefringent element modifies final phase difference between the orthogonal sets of interference fringes resulting in $\Delta \theta_{2} \neq \Delta \theta_{1}$, in general.

In addition, the injected light vector $(E)$ which is typically slightly elliptically polarized at the output end of the sensing fiber, can thus be resolved into its two orthogonal field components, $\mathbf{E}_{x}$ and $\mathbf{E}_{y}$, with an initial phase difference of $\Delta \theta_{1}$. After reflection back from the vibrating target surface, the two components are then re-injected into the fiber and interfered with their respective counterparts reflected off the fiber-air interface of the sensing arm. Two sets of interference signals due to the orthogonal fields are thus generated within a single fiber arm, in effect, leading to a "virtual" dual-cavity interferometer. With no manipulation of their states of polarization [see Fig. 1(a)], the phase difference between the orthogonal interfering components $\left(E_{x}\right.$ and $\mathbf{E}_{y}$ ) remains unchanged at $\Delta \theta_{1}$ after reflection from the moving target, hence, the two sets of fringes are said to be in phase (no phase shift between two fields). Consequently, as illustrated in Fig. 1(b), by inserting a birefringent element along the optical path as a phase retarder between the two 
fields, $\mathbf{E}_{x}$ and $\mathbf{E}_{y}$, on their return paths, a further phase retardation, $\Delta \theta_{2}$ (where $\Delta \theta_{2} \neq \Delta \theta_{1}$ generally), is thereby obtained.

The resulting interference intensities for both the $x$ and $y$ components can thus be simply detected as the sums of their respective fields [10]:

$$
\begin{aligned}
& I_{x}=I_{x r}+I_{x s}+2 \sqrt{I_{x r} I_{x s}} \cos \Delta \theta_{x} \\
& I_{y}=I_{y r}+I_{y s}+2 \sqrt{I_{y r} I_{y s}} \cos \Delta \theta_{y}
\end{aligned}
$$

where $I_{x r}, I_{x s}, I_{y r}$, and $I_{y s}$ are the reference and sensing intensities for both components, respectively. $\Delta \theta_{2}$ $\left(=\Delta \theta_{y}-\Delta \theta_{x}\right)$ is the newly imposed phase difference between the orthogonal signals and can be given by $\left(\theta_{y r}-\theta_{y s}\right)-\left(\theta_{x r}-\theta_{x s}\right)$. When $\Delta \theta_{2}$ is manipulated into a difference of $\lambda / 2$, an optically generated quadrature phase-shift can thereby be obtained.

We therefore have two sets of interference signals [associated with $I_{x}$ and $I_{y}$ in (3) and (4)] which can be detected separately in this case. By oscillating the intended target, a Doppler effect is "seen" by the sensing fiber and can be observed as a phase lag or lead between the two respective interference signals. Retro-reflective surfaces have been used to ensure optimum retro-injection of the sensing beam back into the fiber arm.

However, the proof-of-concept of our proposed technique was then demonstrated by using a program developed in MATLAB for simulating and analyzing the dual optically quadrature phase-shifted interference signals $\left(\mathbf{E}_{x}\right.$ and $\left.\mathbf{E}_{y}\right)$. Consequently, the final demonstrated results were then plotted out as a displacement curve for the sensor with and without optical manipulation of the states of polarization of two orthogonal fields, as clearly illustrated in Fig. 2.

For non-ambiguous measurement, the time difference between the two interference fringes $(\Delta t)$ was used to indicate the target direction, and $\Delta t$ $(>0=$ movement of target away from sensor and $<0=$ target approaching sensor) has been pre-selected to represent the corresponding phase difference (lead or lag) between the first and second channels on the left and right halves of the oscilloscope trace, respectively. In addition, the first channel has been designated as the reference channel for tracking purposes.

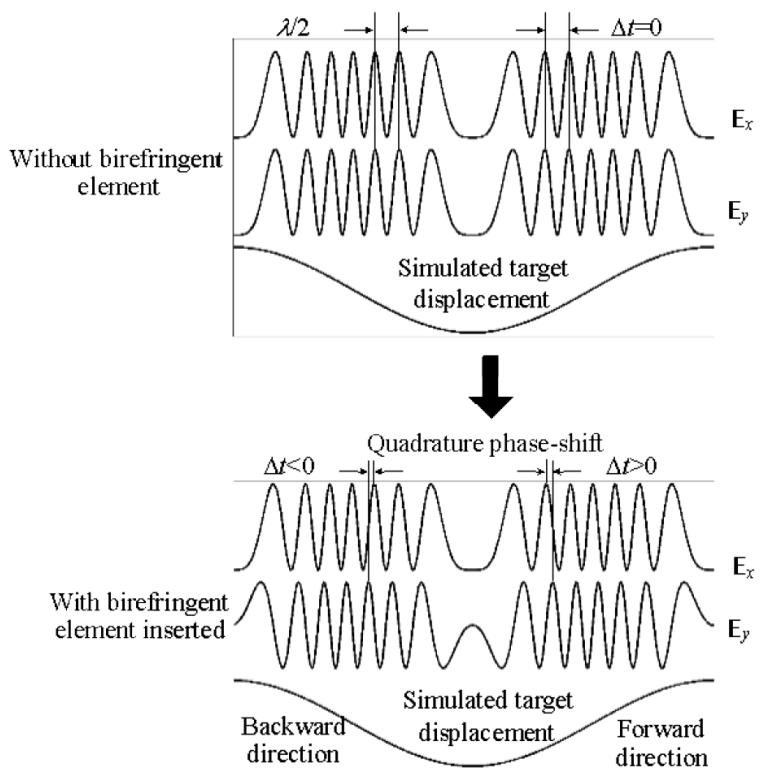

Fig. 2 Simulated output interference signals in the EFFPI configuration without (top) and by inserting the optical birefringent element (bottom) in the optical path.

\subsection{Modified zero-crossing fringe counting technique}

The zero-crossing fringe counting technique has been widely applied in various applications of optical signal processing schemes [18-20] and operates on the principle of an intensity-based analysis method [21]. Each interference fringe is normally sub-divided into one or several levels for locating the points where the intensity from the interference signal crosses the mean intensity value.

In this work, we modified the zero-crossing technique [16] for analyzing two simultaneous interference signals with a resolution of $\lambda / 64$. The output intensities from the two sets of interference fringes were sub-divided into 8 levels by using the "bucket-bin" method [22]. Their respective peaks and valleys were also taken into account to confirm the quadrature condition. The mean intensity $\left(I_{m}\right)$ value for each of the output interference fringes was first identified to allow the location of the point 
where the signal crossed this value and can be given as

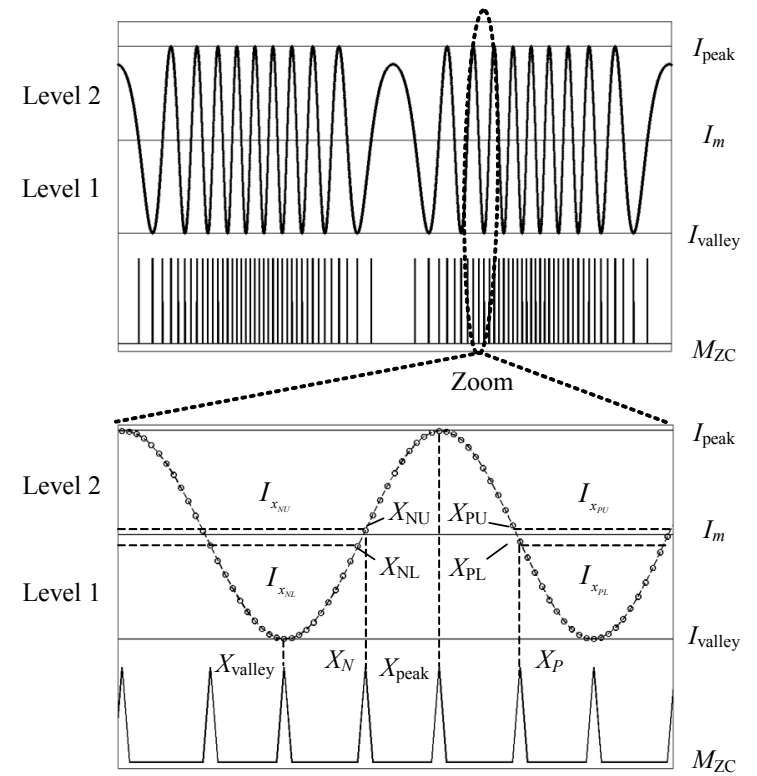

Fig. 3 Principle of the modified zero-crossing fringe counting technique $\left(M_{Z C}\right.$ denotes the modified zero-crossing points).

$$
I_{m}=\frac{I_{\text {peak }}+I_{\text {valley }}}{2}
$$

where $I_{\text {peak }}$ and $I_{\text {valley }}$ represent the maximum and minimum intensity values of the detected interference signal, respectively. The proposed fringe processing technique is thus shown in Fig. 3. We can consider that two zero-crossing points are generated on two sides of the interference fringe to give $X_{N}$ (left position point) and $X_{P}$ (right position point). Moreover, getting each point can thus be divided into lower and upper steps $\left(X_{\mathrm{NU}}\right.$ and $X_{\mathrm{PU}}$ for the upper step and $X_{\mathrm{NL}}$ and $X_{\mathrm{PL}}$ for the lower step, respectively). These are then used to determine the exact crossing-over points, $X_{N}$ and $X_{P}$, respectively, which can be given from

$$
\begin{gathered}
X_{N}=\frac{I_{m}-I\left(X_{\mathrm{NL}}\right)}{I\left(X_{\mathrm{NU}}\right)-I\left(X_{\mathrm{NL}}\right)}+X_{\mathrm{NL}} \\
X_{P}=\frac{I_{m}-I\left(X_{\mathrm{PL}}\right)}{I\left(X_{\mathrm{PL}}\right)-I\left(X_{\mathrm{PU}}\right)}+X_{\mathrm{PL}} .
\end{gathered}
$$

As can be calculated from (6) and (7), modified zero-crossing signals are then obtained at four points $\left(X_{N}, X_{P}, X_{\text {peak }}\right.$, and $\left.X_{\text {valley }}\right)$ in one time period where $X_{\text {peak }}$ and $X_{\text {valley }}$ represent the peak and valley points of one set of interference signal, respectively. Further, by exploiting the imposed relationship of the phase-shift between the two interference signals, the zero-crossing signals can actually be generated at 32 points by sub-dividing the intensity value into 8 levels for each interference fringe via (5), hence, doubling the original resolution for each signal. This technique is, generally, exploiting an increment of resolution of the demodulated signals.

As illustrated in Fig. 4, the output of the modified zero-crossing signal $\left(M_{\mathrm{zc}}\right)$ is obtained at 32 points. These points are next processed by the developed program for plotting the displacement information. Each step of the displacement tracing is, normally, associated to the points that are obtained from the modified zero-crossing signals. Moreover, the program could also be used for determining the direction of the vibrating target by considering the space/gap of the $M_{\mathrm{zc}}$. For example, if there is no modified zero-crossing signal generated during the directional period (see Fig. 4), this implies that the direction of the moving target would be changed. In addition, this technique suffers from no directional ambiguity when the target vibrates due to the two unique but optically phase-shifted in quadrature interference signals.

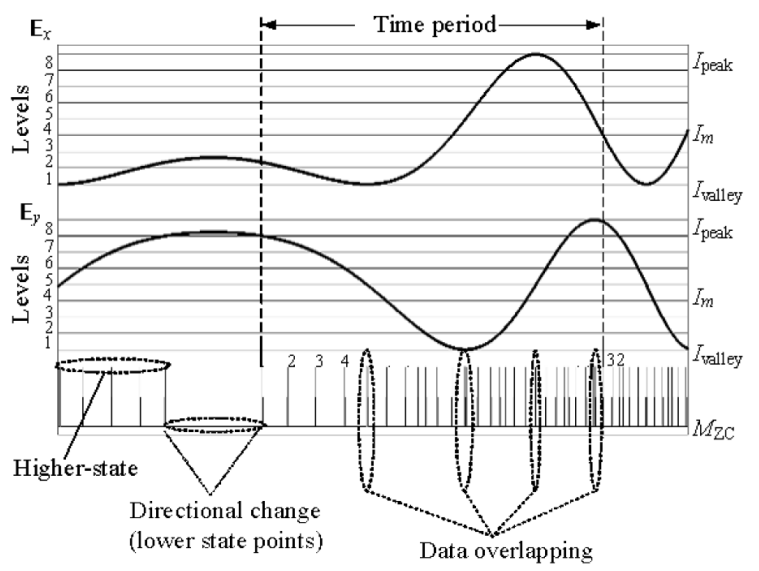

Fig. 4 Zero-crossing signals sub-divided into eight levels to obtain $\lambda / 64$ of resolutions.

Unfortunately, the effect from data overlapping of the output zero-crossings occurrs during fringe processing due to the output points between two 
components being exploited very close to each other's position. This has the consequence that the program could not further distinguish these points. This effect easily contributes to the wrong positional plotting of the displacement curve leading to the measurement errors.

To improve the inherent errors due to data overlapping, we employed zero-phase digital filtering in our algorithm which was written in MATLAB to remove the system noise from the output interference fringes. The algorithm is illustrated in Fig. 5, showing only the major processing steps such as calculating a mean intensity value, counting the number of fringes, plotting the displacement measured, and also determining the target directional movement.

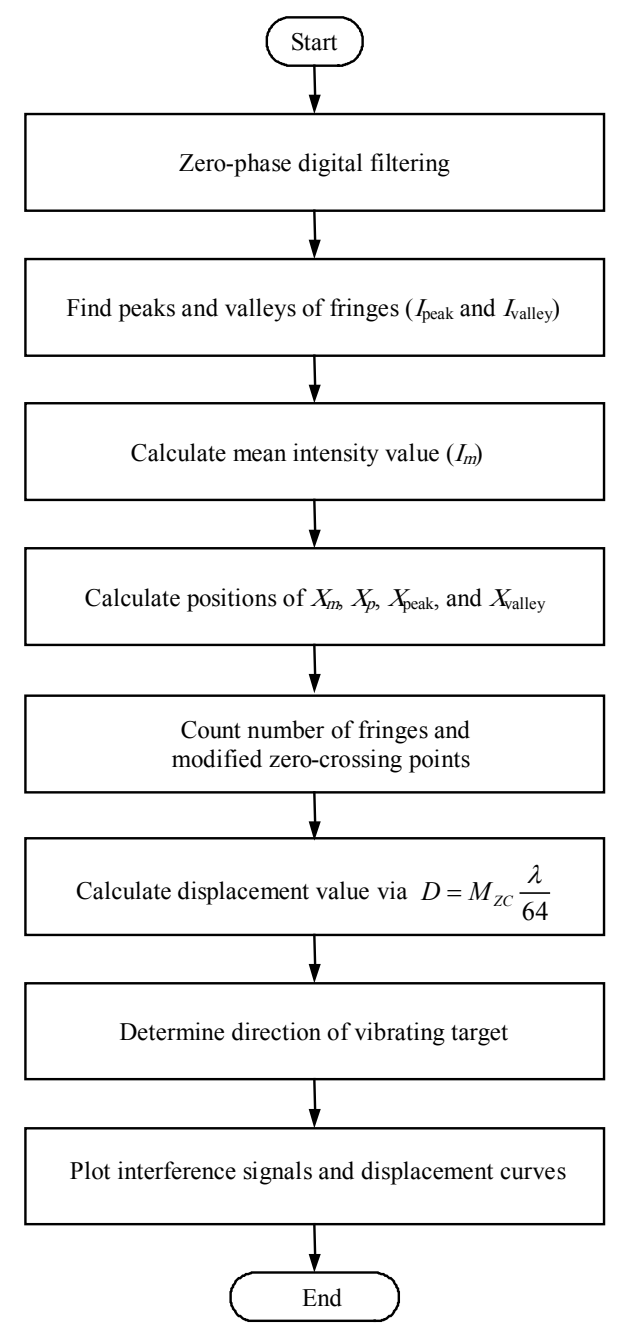

Fig. 5 Block diagram representation of algorithm used in the data processing scheme.

\section{Experimental setup}

The experimental schematic of the extrinsic fiber-based Fabry-Perot interferometer for the dynamic displacement measurement of an oscillating target is illustrated in Fig. 6 .

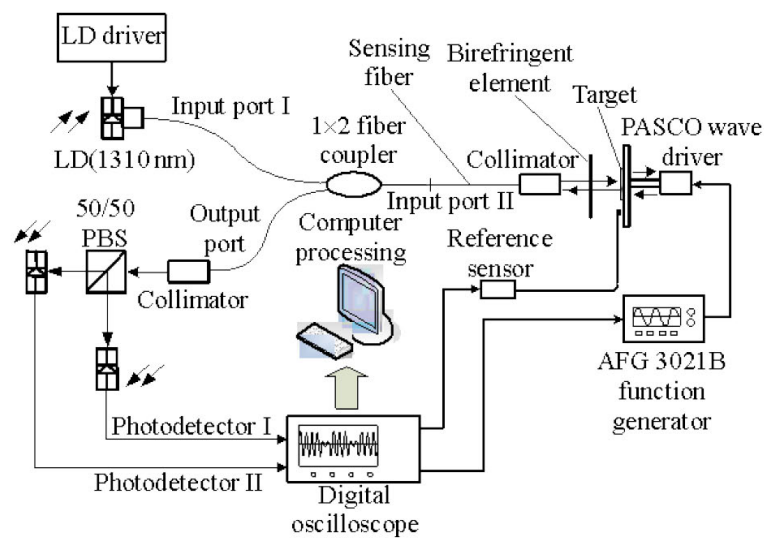

Fig. 6 Experimental configuration of the EFFPI sensor (LD denotes the fiber pigtailed laser diode, and PBS is the polarizing beam splitter).

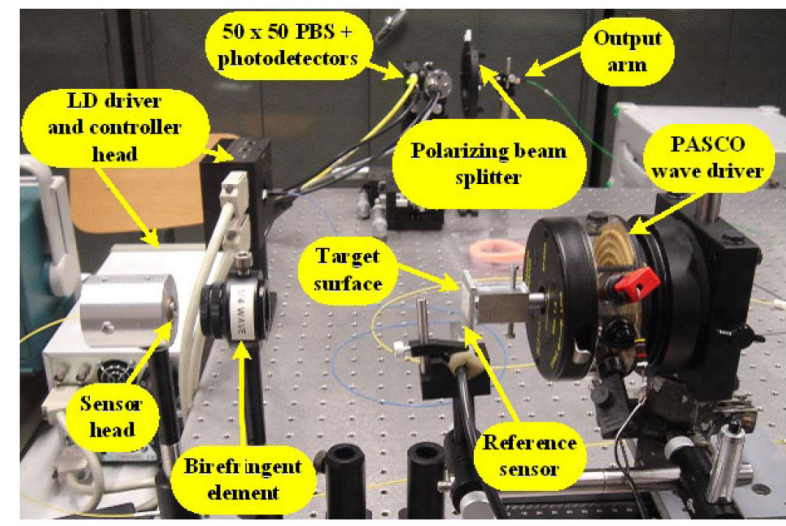

Fig. 7 Photograph of the EFFPI sensor for the dynamic displacement measurement.

The use of a micro-prismatic retro-reflective sheeting on the target surface enabled high flexibility during the sensor-target alignment procedure (from perpendicular incidence up to incident angles of about $30^{\circ}$ ). The sheeting was secured to a mechanical vibrator (PASCO Scientific SF-9324) excited by a classical function generator (Tektronix AFG 3021B) at various excitation amplitudes and frequencies. An image of the interferometer setup is shown in Fig. 7. In order to achieve a "virtual" dual-cavity, a 50/50 polarizing 
beam splitter (PBS) was employed just before two photodetectors to split the re-injected lightwave into its orthogonal components. Hence, two sets of interference signals due to the $\mathbf{E}_{x}$ and $\mathbf{E}_{y}$ waves were generated and made to interfere with their reference pairs reflected off the fiber-air interface at the photodetectors, respectively. The detected interference signals were then displayed on a digital oscilloscope, and the fringes were tracked via a dedicated computer to extract the desired displacement information simultaneously. A commercially-available reference sensor (Philtec D63 displacement sensor with a far-side sensitivity of $-2.786 \mathrm{mV} / \mu \mathrm{m}$ and a far-field noise limit of $80 \mathrm{~nm}$ ) was used as the reference for comparison of the data obtained from the fiber interferometer.

\section{Experimental results and discussion}

To study the performance of the developed interferometer, three different excitation waveforms were used to generate the dynamic movements. The excitation amplitude varied from $0.1 \mathrm{~V}$ to $5 \mathrm{~V}$ but at a fixed excitation frequency of about $125 \mathrm{~Hz}$. The preliminary experimental results obtained showed the ability of our sensor to function under different excitation regimes.

As illustrated in Fig. 8, the two sets of interference signals in quadrature can be clearly seen (Channels 1 and 2, respectively), while Channel 3 indicates the output displacement curve from the reference sensor. Channel 4 shows the sinusoidal excitation waveform transmitted to the target from the function generator. For $500 \mathrm{mV}$ excitation amplitude, a displacement value of $15.793 \mu \mathrm{m}$ was obtained by the reference sensor while our interferometric sensor measured a displacement of $15.769 \mu \mathrm{m}$. This gave a percentage error of $0.15 \%$.

As illustrated in Fig.9, a square wave excitation was next demonstrated for the displacement measurement. With the same excitation amplitude condition, the output displacement of $9.84 \mu \mathrm{m}$ from the reference sensor was exploited, while the

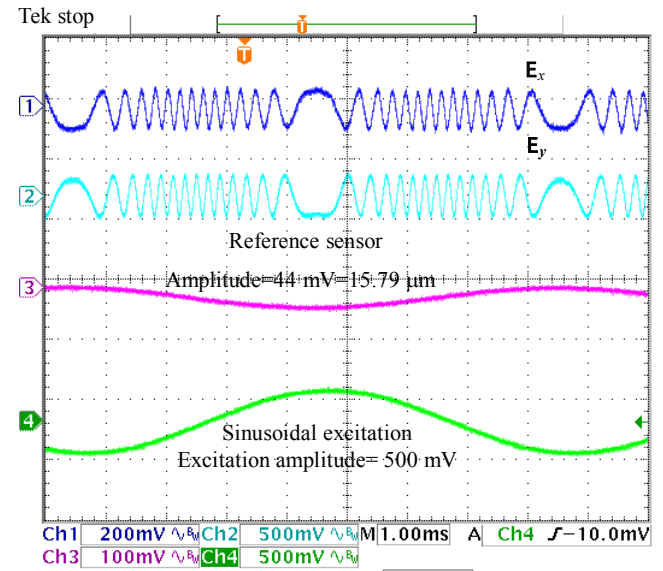

Fig. 8 Output signals from the square wave excitation at an excitation frequency of $125 \mathrm{~Hz}$ and $500 \mathrm{mV}_{\mathrm{p} \text {-p }}$ amplitude.

interferometer gave a displacement of $9.83 \mu \mathrm{m}$, respectively. However, the nature of this waveform suggested very fast falling and rising edges, therefore, the output interference signals should occur over a very short time as well. Unfortunately, the output fringes from the two components can, however, be exploited only over a very short time at the rising and falling periods of the excitation waveform. Furthermore, at the stable-state condition (see in Fig. 9), the interference fringes should not occur, but in the experimental results, fringes were obtained from the fiber interferometer. These phenomenal effects can thus appear because of two possible reasons: 1) the non-zero of the stable-state generated by the classical function generator which drives the signal to the mechanical vibrator, and 2) the out-of-synchronization of the vibrator with respect to the excitation injected (limit between the frequency and amplitude), provoking an unexpected mechanical movement. This last effect has thus resulted in a time delay between the generator and target movement.

The dithering effects resulting from non-zero stable states and also out-of-synchronization of vibrator can, however, be overcome by using the digital high-precision or high-speed function generators instead of the classical function generator device. It causes to several advantages such as the high symmetry, constant sweep width, and high 
stability.

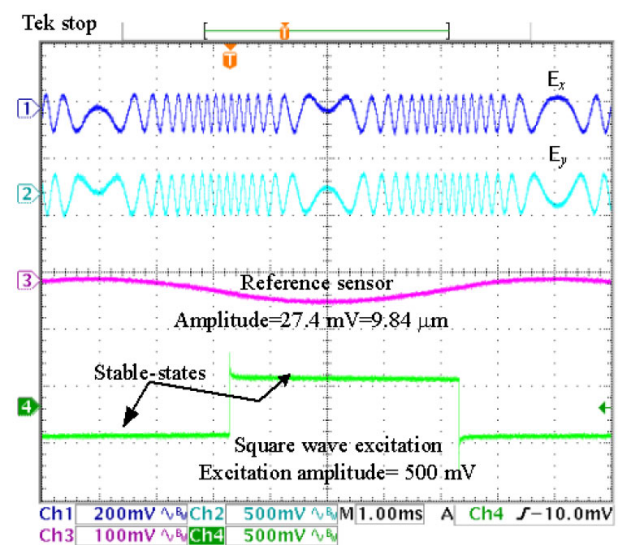

Fig. 9 Output signals from the square wave excitation at an excitation frequency of $125 \mathrm{~Hz}$ and $500 \mathrm{mV}_{\mathrm{p}-\mathrm{p}}$ amplitude.

The triangular excitation waveform, as shown in Fig. 10, had the same excitation conditions $(\sim 125 \mathrm{~Hz}$ of frequency and $500 \mathrm{mV}$ for amplitude excitation). The reference sensor measured a displacement of $12.92 \mu \mathrm{m}$ while a displacement of $12.90 \mu \mathrm{m}$, without any directional ambiguity, was obtained by the interferometer.

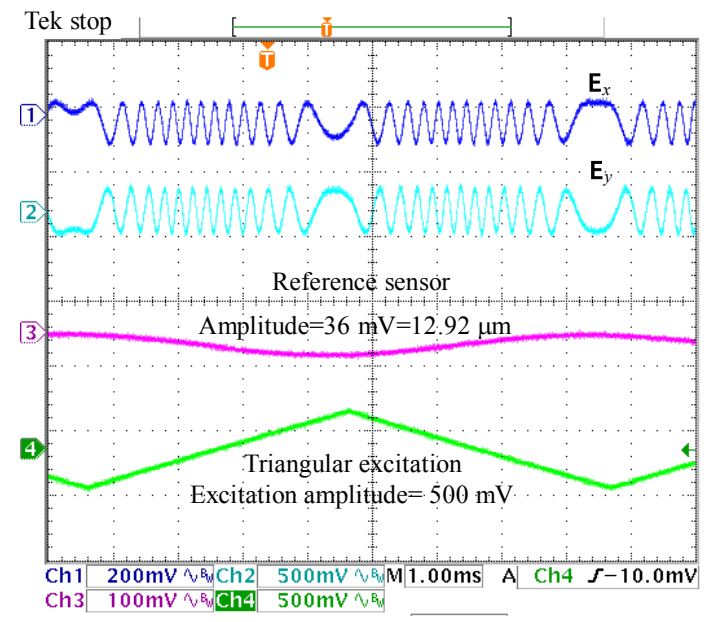

Fig. 10 Output signals from the triangular excitation at an excitation frequency of $125 \mathrm{~Hz}$ and $500 \mathrm{mV}_{\mathrm{p} \text {-p }}$ amplitude.

Essentially, it was found that the quadrature condition was still tightly maintained for all three excitation waveforms, confirming the stability of the optically imposed phase-shift throughout the entire series of experiments. Moreover, for investigating the displacement errors, each measurement was carried out over 20 times at the same excitation amplitude. A displacement range of $0.7 \mu \mathrm{m}-$
$81.65 \mu \mathrm{m}$ was achieved for the sinusoidally varying profile while the average measurement error over the entire dynamic range was estimated as $0.35 \%$ by comparing with the reference data. The lower displacement range corresponds to the ability of the fiber interferometer to measure any half-wavelength amplitude with respect to the laser diode wavelength. Further, the average precision has been calculated in terms of a bi-section of the standard deviation of the actual displacement measured and was found to be about $\pm 0.15 \mu \mathrm{m}$.

For square wave excitation, the displacement range achieved was $0.7 \mu \mathrm{m}-139 \mu \mathrm{m}$ with an average displacement error of $0.2 \%$. The average precision obtained was similar to the sinusoidal profile $(\sim \pm 0.15 \mu \mathrm{m})$ due to the non-zero periods of the rising and falling edges and de-synchronization between the function generator and vibrator as described earlier.

In addition, the triangular displacement profile at a constant frequency of about $125 \mathrm{~Hz}$ but with the varying excitation amplitudes from $0.1 \mathrm{~V}$ to $5 \mathrm{~V}$ was next demonstrated. The displacement range of $0.7 \mu \mathrm{m}-81.65 \mu \mathrm{m}$ measured by our sensor was associated with an average displacement error of $0.2 \%$. We also obtained an average precision of about $\pm 0.1 \mu \mathrm{m}$.

The three sets of displacements corresponding to the excitation signals used are graphically summarized in Fig. 11 together with their associated errors in the form of error bars.

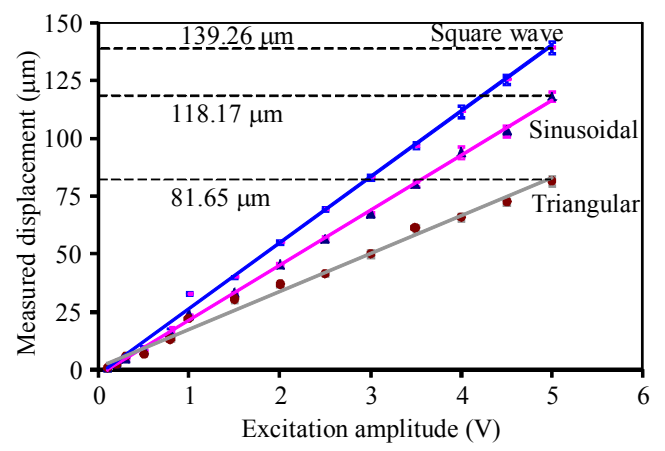

Fig. 11 Summarization of the measured displacements for three different waveforms: square, sinusoidal, and triangular waves. 


\section{Conclusions}

An extrinsic fiber Fabry-Perot interferometer with an optically quadrature condition has been exploited for the dynamic displacement measurement under various excitation waveforms. Three different waveforms with a constant frequency of about $125 \mathrm{~Hz}$ have been studied with the varying amplitudes from $0.1 \mathrm{~V}$ to $5 \mathrm{~V}$. The maximum displacement measured was about $139 \mu \mathrm{m}$. A relatively high resolution of about $20 \mathrm{~nm}$ has been achieved by adopting the modified zero-crossing technique. The interferometer exhibited a good performance with an ability to measure the dynamic displacement under various waveforms or profiles and with relatively high accuracy. Further, the sensor can overcome directional ambiguity, which is a highly significant implication in applications relating to precision mechatronics.

Open Access This article is distributed under the terms of the Creative Commons Attribution License which permits any use, distribution, and reproduction in any medium, provided the original author(s) and source are credited.

\section{References}

[1] D. N. Borza, "Mechanical vibration measurement by high-resolution time averaged digital holography," Measurement Science and Technology, 2005, 16(9): 1853-1864.

[2] H. Tazawa, T. Kanie, and M. Katayama, "Fiber-optic coupler based refractive index sensor and its application to bio-sensing," Applied Physics Letters, 2007, 91(11): 113901-133905.

[3] F. T. S. Yu and S. Yin, Fiber optic sensors. New York: Marcel Dekker Inc., 2002.

[4] E. Udd and W. B. S. Jr, Fiber optic sensor: an introduction for engineers and scientists. New York: John Wiley \& Sons Inc., 1991.

[5] H. C. Seat and S. Pullteap, "An extrinsic fiber fabry-perot interferometer for dynamic displacement measurement," in IEEE International Conference on Mechatronics and Automation (ICMA), Harbin, pp. 3025-3030, 2007.

[6] K. Kesavan, K. Ravisankar, S. Parivallal, P. Sreeshylam, and S. Sridhar, "Experimental studies on fiber optic sensors embedded in concrete,' Measurement, 2010, 43(2): 157-163.

[7] B. Regez, M. Sayeh, A. Mahajan, and F. Figueroa, “A novel fiber optics based method to measure very low strains in large scale infrastructures," Measurement, 2009, 42(2): 183-188.

[8] S. Pullteap, "Development of an optical fiber based interferometer for small vibration measurement," in IEEE International Conference on Optical Communication and Network (ICOCN), Chonburi, pp. 107-110, 2012.

[9] P. Antunes, A. M. Rocha, H. Lima, H. Varum, and P. S. Andre, "Thin bonding wires temperature measurement using optical fiber sensors," Measurement, 2011, 44(3): 554-558.

[10] H. C. Seat, S. Pullteap, and T. Bosch, "An extrinsic fibre optic interferometer with possible signal fading compensation for vibrometric applications," in Proceedings of the IEEE Instrumentation and Measurement Technology Conference, Ottawa, pp. 2236-2241, 2005.

[11] X. Zhou and Q. Yu, "Wide-range displacement sensor based on fiber-optic fabry-perrot interferometer for subnanometer measurement," IEEE Sensors Journal, 2011, 11(7): 1602-1606.

[12] A. Khiat. F. Lamarque, C. Prelle, P. Pouille, M. L. Schadel, and S. Buttgenbach, "Two-dimension fiber optic sensor for high-resolution and long-range linear menaurements," Sensors and Actuators A: Physical, 2010, 158(1): 43-50.

[13] R. P. Tatam, "Applied optics to engineering photonics: a retrospective," Photonic sensors, 2011, 1(4): 295-322.

[14] B. Chen, J. Luo, and D. Li, "Code counting of optical fringes: methodology and realization," Applied Optics, 2005, 44(2): 217-223.

[15] T. K. Gangopadhyay, "Non-contact vibration measurement based on an extrinsic Fabry-Perot interferometer implemented using arrays of single-mode fibres," Measurement Science and Technology, 2004, 15(5): 911-917.

[16] S. Pullteap, H. C. Seat, and T. Bosch, "Modified fringe-counting technique applied to a dual-cavity fiber Fabry-Pérot vibrometer," Optical Engineering, 2007, 46(11): 115603-1-115603-8.

[17] G. Hernandez, Fabry-perot interferometers. Cambridge: Cambridge University Press, 1986.

[18] M. Norgia and C. Svelto, "Novel measurement method for signal recovery in optical vibrometer," IEEE Transactions on Instrumentation and Measurement, 2008, 57(8): 1703-1707. 
[19] S. Topcu, L. Chassagne, D. Haddad, and Y. Alayli, "Heterodyne interferometric technique for displacement control at the nanometric scale," Review of Scientific Instruments, 2003, 74(11): 4876-4880.

[20] Y. Zhu, J. D. Zuegel, J. R. Marciante, and H. Wu, "Distributed waveform generator: a new circuit technique for ultra-wideband pulse generation, shaping and modulation," IEEE Journal of Solid-State Circuits, 2009, 44(3): 808-823.

[21] K. J. Gàsvik, Optical Metrology. London: Wiley \& Sons., 2002.

[22] K. J. Gàsvik, K. G. Robbersmyr, and T. Vadseth, "Fringe location by means of a zero-crossing algorithm," in Proc. SPIE, vol. 1163, pp. 64-70, 1989. 